Research Article

\title{
Analysis of the Vibration of the Ground Surface by Using the Layered Soil: Viscoelastic Euler Beam Model due to the Moving Load
}

\author{
Hong-Yuan Huang $\mathbb{D}^{1},{ }^{1}$ Ming-Jie Zhao ${ }^{\mathbb{D}},{ }^{1}$ Yao Rong $\mathbb{D}^{2},{ }^{2}$ Yang Sun $\mathbb{D}^{2},{ }^{2}$ and Xiao Xiao $\mathbb{i}^{3,4}$ \\ ${ }^{1}$ Engineering Research Center of Diagnosis Technology of Hydro-construction of Chongqing, Chongqing Jiaotong University, \\ Chongqing 400074, China \\ ${ }^{2}$ Jiangxi Transportation Research Institute, Nanchang 330200, China \\ ${ }^{3}$ School of Civil and Architecture Engineering, Nanchang Institute of Technology, Nanchang 330099, China \\ ${ }^{4}$ Jiangxi Provincial Engineering Research Center of the Special Reinforcement and \\ Safety Monitoring Technology in Hydraulic \& Civil Engineering, Nanchang 330099, China
}

Correspondence should be addressed to Xiao Xiao; xiaoxiao8808@163.com

Received 30 November 2020; Revised 30 December 2020; Accepted 25 January 2021; Published 8 February 2021

Academic Editor: Xinyu Xie

Copyright $\odot 2021$ Hong-Yuan Huang et al. This is an open access article distributed under the Creative Commons Attribution License, which permits unrestricted use, distribution, and reproduction in any medium, provided the original work is properly cited.

\begin{abstract}
Moving loads will have a certain impact on the safety of the structures. Since concrete is a viscoelastic material, the elastic concrete model cannot describe its viscoelastic characteristics under moving loads. It is necessary to establish a model that can describe the viscoelastic characteristics of concrete materials. In addition, the layered of the soil is also an important factor affecting the propagation of subway vibration waves. Considering the effects of the properties of the concrete material of the subway tunnel structure and the layered soil foundation as well as the load velocity on the vibration of the ground surface caused by the moving load, the standard linear elastic solid Euler beam model is described for the subway tunnel structure in this paper. The equivalent stiffness of the layered soil-viscoelastic beam coupling system subjected to a moving load is formed by using the transmission and reflection matrix (TRM) method. The numerical solution of ground surface displacement caused by subway tunnel in time-space domain is obtained by IFFT algorithm. The correctness of the algorithm is verified by comparing with the reference results. Numerical results show that, with the increase of the viscous coefficient of the viscoelastic Euler beam, the vibration amplitude of the ground surface will decrease. Up to a certain value of the increasing the viscous coefficient of the Euler beam, it will have little effect on the vibration amplitude of the ground surface. Therefore, the standard solid model of viscoelastic Euler beam can well describe the creep and relaxation of materials. The model of viscoelastic beam is reasonable for the working condition of subway tunnel concrete structure. At high speed of moving load, the maximum value of ground surface displacement spectrum will appear at the smaller frequency domain and the maximum value of displacement spectrum will also increase for the soft layer soil, while it is opposite to that of the stiffer layer soil.
\end{abstract}

\section{Introduction}

Vibration induced by subway traffic is a major concern for civil engineers as it causes annoyance to residents or even damage to adjacent structures. Generally, analytical method and numerical method are applied in this area [1-5]. Yang and Hung [6] used 2.5D FEM to study the ground vibrations caused by moving loads in the circle lining tunnel. Bian et al.
$[7,8]$ proposed a train-track-foundation coupled model to analyse the interaction between tunnel structure and surrounding soil using 2.5D FEM. The results show that transferring band width of ground wave is becoming narrow while frequency of moving loads increasing. Also, by means of the consistency or periodicity along with the tunnel axis in the dynamic system of tunnel-free wave field, the dynamic response solution subjected to a moving load is studied by 
Liu et al. [9]. Gupta and Degrande [10] employed Floquet transform with a periodic coupled model of track and tunnel-soil system to analyse the vibration isolation effect of continuous and discontinuous floating slab tracks. Al-Furjan et al. [11] presented the influences of some geometrically and physically parameters of multiscale hybrid nanocomposite and new smart and composite materials fiber on the frequency of the sandwich structure. Al-Furjan et al. [12] adopted a mathematical derivation to develop a nonlinear dynamic model for the nonlinear frequency and chaotic responses of the multiscale hybrid nanocomposite reinforced disk in the thermal environment and subject to a harmonic external load. Metrikine and Vrouwenvelder [13] developed an analytical solution for the tunnel in twodimensional elastic-layered foundation subjected to a moving point load. The research results show that when the train speed is close to the Rayleigh wave velocity of the foundation soil, the vibration level of the track-ground structure will increase significantly. Usually, the velocity of Rayleigh wave on soft soil foundation is about $200 \mathrm{~km} / \mathrm{h}$, and the current high-speed trains can easily exceed this speed. Therefore, the vibration of the soil layer caused by the moving load of the subway tunnel is very important.

As we know, natural soil with different deposition ages are always layered form, so the layered soil foundation model is closer to the actual engineering. There are a variety of analytical and numerical methods to solve the dynamic response of a layered half-space [14]. Tomson [15] and Harkrider [16] formed and developed the transfer matrix method of layered soil based on the finite element thin layer method. Rajapakse and Senjuntichai $[17,18]$ proposed an accurate stiffness matrix method for the dynamic response of layered soils using integral transformation. Al-Furjan et al. [12] develop a nonlinear dynamic model for the nonlinear frequency and chaotic responses of the multiscale hybrid nanocomposite reinforced disk in the thermal environment and subject to a harmonic external load. Luco and Apsel [19] used the TRM method to obtain the dynamic response Green function of the three-dimensional layered elastic halfspace. Xu et al. [20] applied the TRM method to the dynamic response of an infinite Euler beam on a layered saturated half-space soil subjected to a moving load. Judging from the existing research results, the TRM method has high accuracy in overcoming the matrix ill-conditioned problems caused by dynamic response of high-frequency or large differences between layered soils, etc., and it has obtained wide range of applications in the dynamic response of layered soils.

The subway tunnel structure is generally polymer concrete. It not only has elastic deformation but also has viscous deformation, which can generate damping in the process of energy loss so called a kind of viscoelastic material. Viscoelastic damping materials have a certain control effect on structural vibration, and more and more attention been paid on its vibration analysis problems by experts and scholars. Chen et al. [21] used the standard rheological model (STD) to establish kinetic equations of viscoelastic structures in the time domain and Lagrangian domain and analyzed the dynamic problems of viscoelastic structures. $\mathrm{Gu}$ et al. [22] and Liu et al. [23] utilized static experiments to analyse the constitutive relationship of viscoelastic materials and deduced the constitutive equations of viscoelastic materials in the complex number domain, time domain, and frequency domain. Then, the dissipation energy of viscoelastic damping materials was investigated by means of the elastic constitutive equation. Surie and Cederbaum [24] derived the constitutive equation of viscoelastic material based on Boltzmann's principle and analyzed the basic dynamic model of inelastic beam. Argyris et al. [25] analyzed the chaotic motion of viscoelastic beams by using the constitutive relationship of differential viscoelastic materials. Chen et al. $[26,27]$ established partial differential-integral equations for nonlinear viscoelastic moving beams, which were used to illustrate the dynamic model of geometric nonlinear viscoelastic beams and simplified the equations. Marynowski and Kapitaniak [28] studied the dynamic characteristics of a stable moving beam on the basis of the viscoelastic constitutive model. Li et al. [29] extended several typical dynamic models such as complex modulus model, fractional and exponential model, fractional and derivative model, and micro-oscillator model focusing on its application. AlFurjan et al. [30] deal with the frequency analysis of imperfect honeycomb core sandwich disk with multiscale hybrid nanocomposite (MHC) face sheets rested on an elastic foundation. Moreover, Li et al. [31] analyzed the amplitude-frequency response of the control model for forced vibration of the nonlinear viscoelastic Timoshenko beam in the steady state, and then the numerical simulation was used to investigate the influence of the damping of viscoelastic materials and external effects on the stable state of the structure. The above research results provide a good theoretical basis for viscoelastic damping materials to be used in structural vibration control.

The above literatures have adopted different methods to study the response of layered soil under dynamic loads. However, the research concerning a layered half-space has been mainly restricted to the elastic case and the dynamic response of an infinite beam on a layered poroelastic halfspace to moving loads is seldom studied in the literature. In addition, since concrete is a viscoelastic material, the elastic concrete model cannot describe its viscoelastic characteristics under moving loads. It is necessary to establish a viscoelastic model that can describe the viscoelastic characteristics of concrete materials. In this paper, the layered soil-viscoelastic Euler beam model subjected to a moving load is used to analyse the surface vibration characteristics caused by subway tunnels in the layered soil. The effects of structural damping on vibration are described by a standard linear elastic solid model for the subway tunnel structure. And then, the equivalent stiffness of the layered foundation is formed by the TRM method. The numerical solution of the vibration displacement with respect to the soil surface in the time-space domain is obtained by the IFFT algorithm. The elastic modulus, viscoelastic damping coefficient of the viscoelastic Euler beam, and the influence of layered soil on the vibration of the soil surface are also calculated and analyzed by means of the calculation model in this paper. At last, the calculation method in the article is compared with the existing results to verify the correctness of the algorithm. 
The analysis of the calculation example shows that the subway tunnel structure described by the viscoelastic beam model behaves plasticity. The reason is that the shear modulus changes with the calculation frequency within a certain frequency range, but after the calculation frequency increases to a certain value, the shear modulus will not change with the frequency. Therefore, the standard solid model of viscoelastic beam can better describe the creep and relaxation of the material.

\section{Computation Model and Governing Equations for the Subway Tunnel Structure in the Layered Soil Foundation}

For the subway tunnel structure in the layered soil foundation, a simplified two-dimensional physical model is used, as shown in Figure 1. Concerning the viscoelasticity of the subway tunnel structure made of concrete material and the layered soil foundation, the subway tunnel with a buried depth of $h$ is simplified to an infinite length viscoelastic Euler beam structure. The thickness of each layer of soil is $h_{i}$, and the total thickness of the system is $(h+H)$. The subway tunnel is subjected to the vertical moving load of the load concentration $F_{z}$ and velocity $v_{c}$.

The soil is treated with an elastic medium with viscoelastic characteristics. The motion equation and constitutive relationship in the form of displacement can be written as

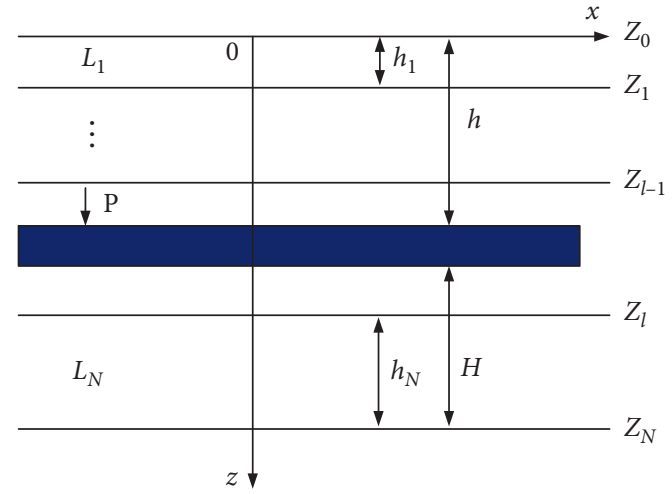

FIGURE 1: A schematic illustration of the layer soil-viscoelastic beam coupling system subjected to a moving load.

$$
\begin{aligned}
\hat{\mu} u_{i, j j}+(\bar{\lambda}+\widehat{\mu}) u_{j, j i} & =\rho \ddot{u}_{i}, \\
\sigma_{i j} & =2 \widehat{\mu} \varepsilon_{i j}+\widehat{\lambda} \delta_{i j} e,
\end{aligned}
$$

where $\overline{\bar{\lambda}} \frown=\lambda+\lambda^{*}(\partial / \partial t), \hat{\mu}=\mu+\mu^{*}(\partial / \partial t), \lambda$ and $\mu$ are Lame constants, $u_{i}(i=1,(2)$ is the displacement of soil skeleton, $\sigma_{i j}$ is the stress of bulk material, $\varepsilon_{i j}$ denotes the strain tensor of the solid skeleton, and $\rho$ and $e$ represent the bulk density of the porous medium and the volumetric stain, respectively.

The vibration equation of the subway tunnel structure is as follows:

$$
\rho_{B} \ddot{W}_{b}+E_{b} I_{b} \frac{\partial^{4} \mathbf{W}_{b}}{\partial x^{4}}=F z \delta\left(x-v_{c} t\right)+a\left(\sigma_{z z}\left(x, h^{-}, t\right)-\sigma_{z z}\left(x, h^{+}, t\right)\right),
$$

where $\rho_{B}, W_{b}, E_{b}$, and $I_{b}$ represent the density, vertical displacement, shear modulus, and polar moment of inertia for the subway tunnel structure, respectively; $F_{z}$ denotes the load amplitude; and $a$ is the average diameter of tunnel structure.

For an infinite length viscoelastic Euler beam, the standard linear solid model theory is used to describe its damping. The complex elastic modulus of the viscoelastic beam is given by

$$
\begin{aligned}
E_{b} & =E_{b R}\left(\frac{1+i \omega \tau_{b \varepsilon}}{1+i \omega \tau_{b \sigma}}\right), \\
E_{b R} & =\frac{E_{b}^{(1)} E_{b}^{(2)}}{E_{b}^{(1)}+E_{b}^{(2)}}, \\
\tau_{b \sigma} & =\frac{\eta}{E_{b}^{(1)}+E_{b}^{(2)}}, \\
\tau_{b \varepsilon} & =\frac{\eta}{E_{b}^{(2)}}
\end{aligned}
$$

where $E_{b}^{(1)}$ and $E_{b}^{(2)}$ represent elastic parameters of viscoelastic beam, respectively, and $\eta$ is the coefficient of viscosity.

Assuming that the displacement of the subway tunnel in the horizontal direction is zero and the displacement between the subway tunnel and the soil layer in the vertical direction is continuous, the boundary conditions are as follows:

$$
\begin{gathered}
u\left(x, h^{-}, t\right)=u\left(x, h^{+}, t\right)=0, \\
w\left(x, h^{-}, t\right)=w\left(x, h^{+}, t\right)=W_{b}(x, t) .
\end{gathered}
$$

The surface and bottom of layered soil have the following stress and displacement boundary conditions:

$$
\begin{gathered}
\sigma_{z z}(x, 0, t)=\sigma_{z x}(x, 0, t)=0, \\
u(x, h+H, t)=w(x, h+H, t)=0 .
\end{gathered}
$$

\section{Fundamental Solution and the TRM Method}

3.1. Fundamental Solutions in Frequency Wave-Number Domain. For the governing equation of elastic soil, the 
displacement and stress vector of soil can be decomposed by Helmholtz's potential functions ( $\Phi$ and $\psi$ ) as follows:

$$
\begin{aligned}
u & =\frac{\partial \Phi}{\partial x}+\frac{\partial \psi}{\partial z}, \\
w & =\frac{\partial \Phi}{\partial z}-\frac{\partial \psi}{\partial x}, \\
\sigma_{z z} & =\hat{\lambda}\left(\frac{\partial^{2} \Phi}{\partial x^{2}}+\frac{\partial^{2} \Phi}{\partial z^{2}}\right)+2 \widehat{\mu}\left(\frac{\partial^{2} \Phi}{\partial z^{2}}-\frac{\partial^{2} \Phi}{\partial x \partial z}\right), \\
\sigma_{z x} & =\tilde{\mu}\left(2 \frac{\partial^{2} \Phi}{\partial x \partial z}-\frac{\partial^{2} \psi}{\partial x^{2}}+\frac{\partial^{2} \psi}{\partial z^{2}}\right) .
\end{aligned}
$$

Thus, the equations of motion for soil can be decoupled into two wave equations as follows:

$$
\begin{aligned}
\frac{\partial^{2} \Phi}{\partial t^{2}}-\left(c_{L}^{2}+\frac{\lambda^{*}+2 \mu^{*}}{\rho} \frac{\partial}{\partial t}\right)\left(\frac{\partial^{2} \Phi}{\partial x^{2}}+\frac{\partial^{2} \Phi}{\partial z^{2}}\right) & =0, \\
\frac{\partial^{2} \psi}{\partial t^{2}}-\left(c_{T}^{2}+\frac{\mu^{*}}{\rho} \frac{\partial}{\partial t}\right)\left(\frac{\partial^{2} \psi}{\partial x^{2}}+\frac{\partial^{2} \psi}{\partial z^{2}}\right) & =0,
\end{aligned}
$$

where $c_{L}=\sqrt{(\lambda+2 \mu) / \rho}$ and $c_{T}=\sqrt{\mu / \rho}$ represent compression wave velocity and shear wave velocity of soil, respectively.

The Fourier transform with respect to time and space is defined as follows:

$$
\begin{aligned}
\widetilde{\bar{f}}\left(k_{x}, \omega\right) & =\int_{-\infty}^{\infty} \int_{-\infty}^{\infty} f(x, t) e^{-i\left(\omega t-k_{x} x\right)} \mathrm{d} x \mathrm{~d} t, \\
f(x, t) & =\frac{1}{2 \pi} \int_{-\infty}^{\infty} \int_{-\infty}^{\infty} \widetilde{\bar{f}}\left(k_{x}, \omega\right) e^{-i\left(\omega t-k_{x} x\right)} \mathrm{d} k_{x} \mathrm{~d} \omega .
\end{aligned}
$$

Equation (7) can be obtained by the Fourier transform as follows:

$$
\begin{aligned}
&-\omega^{2} \tilde{\bar{\Phi}}-\left(c_{L}^{2}-i \omega \frac{\lambda^{*}+2 \mu^{*}}{\rho}\right)\left(-k_{x}^{2} \tilde{\bar{\Phi}}+\frac{\partial^{2} \tilde{\bar{\Phi}}}{\partial z^{2}}\right)=0, \\
&-\omega^{2} \tilde{\bar{\psi}}-\left(c_{T}^{2}-i \omega \frac{\mu^{*}}{\rho}\right)\left(-k_{x}^{2} \tilde{\bar{\psi}}+\frac{\partial^{2} \tilde{\bar{\psi}}}{\partial z^{2}}\right)=0 .
\end{aligned}
$$

Likewise, the vibration equation of subway tunnel beams in frequency wave-number domain is given by

$$
\begin{aligned}
\left(E_{b} I_{b} k_{x}^{4}-\rho_{B} \omega^{2}\right) \tilde{\bar{W}}_{b}= & 2 \pi F z \delta\left(\omega-k_{x} v_{c}\right)+a\left(\tilde{\bar{\sigma}}_{z z}\left(k_{x}, h^{-}, \omega\right)\right. \\
& \left.-\widetilde{\bar{\sigma}}_{z z}\left(k_{x}, h^{+}, \omega\right)\right) .
\end{aligned}
$$

It can be deduced by Equation (9) as follows:

$$
\begin{aligned}
& \tilde{\bar{\Phi}}=A_{1} e^{\gamma_{1} z}+A_{2} e^{-\gamma_{1} z}, \\
& \widetilde{\bar{\psi}}=A_{3} e^{\gamma_{2} z}+A_{4} e^{-\gamma_{2} z},
\end{aligned}
$$

where $A_{i}(i=1,2,3,4)$ can be determined by the boundary conditions: $\quad \gamma_{1}=\sqrt{\left(k_{x}^{2}-\omega^{2}\right) /\left(\left(c_{L}^{2}-i \omega\left(\lambda^{*}+2 \mu^{*}\right)\right) / \rho\right)}$ and $\gamma_{2}=\sqrt{\left(k_{x}^{2}-\omega^{2}\right) /\left(\left(c_{T}^{2}-i \omega \mu^{*}\right) / \rho\right)}$.

The displacement and stress in the frequency wavenumber domain have the following expressions:

$$
\begin{aligned}
& \tilde{\bar{u}}=i k_{x} \tilde{\bar{\Phi}}+\frac{\partial \tilde{\bar{\psi}}}{\partial z}, \\
& \widetilde{\bar{w}}=\frac{\partial \widetilde{\bar{\Phi}}}{\partial z}-i k_{x} \tilde{\bar{\psi}} \\
& \tilde{\bar{\sigma}}_{z z}=\left(\lambda-i \omega \lambda^{*}\right)\left(-k_{x}^{2} \tilde{\Phi}+\frac{\partial^{2} \tilde{\Phi}}{\partial z^{2}}\right) \\
& +2\left(\mu-i \omega \mu^{*}\right)\left(\frac{\partial^{2} \tilde{\Phi}}{\partial z^{2}}-i k_{x} \frac{\partial \tilde{\bar{\psi}}}{\partial z}\right), \\
& \tilde{\bar{\sigma}}_{z x}=\left(\mu-i \omega \mu^{*}\right)\left(2 i k_{x} \frac{\partial \tilde{\bar{\Phi}}}{\partial z}+k_{x}^{2} \tilde{\bar{\psi}}+\frac{\partial^{2} \tilde{\bar{\psi}}}{\partial z^{2}}\right) .
\end{aligned}
$$

Substituting Equation (2) into Equations (13a) and (13b) and inversion of the Fourier transform give the frequency domain fundamental solution.

\subsection{Equivalent Stiffness of Layered Soil-Beam System Based on} TRM Method. It can be seen from the fundamental solution that for the stress and displacement solutions in the frequency wave-number domain of the $L_{j}$ layer of soil, the unknown coefficients $a, c, b$, and $d$ in the general solution can be replaced by $a^{j} e^{-\gamma_{1}^{j} z_{j}}, c^{j} e^{-\gamma_{2}^{j} z_{j}}, b^{j} e^{\gamma_{1}^{j} z_{j-1}}$, and $d^{j} e^{\gamma_{2}^{j} z_{j-1}}$ in the Cartesian coordinate system (Figure 1), where $z_{j}$ and $z_{j-1}$ are the coordinates of the upper and lower surface of layer $L_{j}$. A large $4 N \times 4 N$ matrix will be formed in using the traditional transfer matrix methods, which will inevitably lead to the matrix ill-conditioned problems for the dynamic response of high-frequency, thereby affecting the accuracy of the calculation results.

Using the TRM method, the transmission and transmission waves of each layer of the soil can be expressed by the soil waves subjected to a moving load, so as to avoid solving large linear equations and make the calculation results more accurately. The frequency wave-number domain solution for the layer $L_{j}$ can be written as follows by means of matrix $\psi^{j}(\xi, z, \omega)$ :

$$
\begin{aligned}
& \boldsymbol{\Psi}^{j}(\xi, z, \omega)_{6 \times 1}=\left[\begin{array}{cccc}
\tilde{\bar{u}}_{x}^{j} & \tilde{\bar{u}}_{z}^{j} & \tilde{\bar{\sigma}}_{x z}^{j} & \tilde{\bar{\sigma}}_{z z}^{j}
\end{array}\right]^{T}, \\
& \boldsymbol{\Psi}^{j}(\xi, z, \omega)_{6 \times 1}=\left[\begin{array}{cc}
\mathbf{D}_{\mathbf{d}}^{j} & \mathbf{D}_{\mathbf{u}}^{j} \\
\mathbf{S}_{\mathbf{d}}^{j} & \mathbf{S}_{\mathbf{u}}^{j}
\end{array}\right]\left[\begin{array}{ll}
\mathbf{W}_{\mathbf{d}}^{j}(z)^{T} & \mathbf{W}_{\mathbf{u}}^{j}(z)^{T}
\end{array}\right]^{T}, \\
& \mathbf{W}_{d}^{j}(\xi, z, \omega)=\left[b^{j} e^{-\gamma_{1}^{j}\left(z-z_{j-1}\right)} d^{j} e^{-\gamma_{2}^{j}\left(z-z_{j-1}\right)}\right] \text {, } \\
& \mathbf{W}_{u}^{j}(\xi, z, \omega)=\left[a^{j} e^{\gamma_{1}^{j}\left(z-z_{j}\right)} c^{j} e^{\gamma_{2}^{j}\left(z-z_{j}\right)}\right]^{T},
\end{aligned}
$$


where $\operatorname{Re}\left(\gamma_{i}^{j} \geq 0,(i=1,2)\right), \quad \mathbf{D}_{\mathbf{d}}^{j}(\xi, \omega)_{2 \times 2}, \quad \mathbf{D}_{\mathbf{u}}^{j}(\xi, \omega)_{2 \times 2}$, $\mathbf{S}_{\mathbf{d}}^{j}(\xi, \omega)_{2 \times 2}$, and $\mathbf{S}_{\mathbf{u}}^{j}(\xi, \omega)_{2 \times 2}$ are represented by $\mathbf{D}_{\mathbf{d}}^{j}=$ $\left[\begin{array}{cc}i k_{x} & -\gamma_{2}^{j} \\ -\gamma_{1}^{j} & -i k_{x}\end{array}\right], \mathbf{D}_{\mathbf{u}}^{j}=\left[\begin{array}{cc}i k_{x} & \gamma_{2}^{j} \\ \gamma_{1}^{j} & -i k_{x}\end{array}\right], \quad \mathbf{S}_{\mathbf{d}}^{j}=\left[\begin{array}{cc}-2 i k_{x} \gamma_{1}^{j} & \gamma^{j} \\ \gamma^{j} & 2 i k_{x} \gamma_{2}^{j}\end{array}\right]$, and $\mathbf{S}_{\mathbf{u}}^{j}=\left[\begin{array}{cc}2 i k_{x} \gamma_{1}^{j} & \gamma^{j} \\ \gamma^{j} & 2 i k_{x} \gamma_{2}^{j}\end{array}\right]$, respectively; $\gamma^{j}=\left(2 k_{x}^{2}-\omega^{2}\right) /$ $\left(\left(C_{T}^{2}-i \omega \mu^{*}\right) / \rho\right)$.

The unknown coefficients of the layer $L_{j}$ can be represented by the down going and up going wave vectors in this layer according to Equation (14), respectively:

$$
\left.\begin{array}{l}
\mathbf{W}_{\mathbf{d}}^{j}\left(\xi, z_{j-1}, \omega\right)=\left[\begin{array}{ll}
b^{j}(\xi, \omega) & d^{j}(\xi, \omega)
\end{array}\right]^{T}, \\
\mathbf{W}_{\mathbf{u}}^{j}\left(\xi, z_{j}, \omega\right)=\left[\begin{array}{ll}
a^{j}(\xi, \omega) & c^{j}(\xi, \omega)
\end{array}\right]^{T}, \\
\mathbf{W}_{\mathbf{d}}^{j}(\xi, z, \omega)=\mathbf{E}^{j}\left(\xi, z-z_{j-1}, \omega\right) \mathbf{W}_{\mathbf{d}}^{j}\left(\xi, z_{j-1}, \omega\right), \\
\mathbf{W}_{\mathbf{u}}^{j}(\xi, z, \omega)=\mathbf{E}^{j}\left(\xi, z_{j}-z, \omega\right) \mathbf{W}_{\mathbf{u}}^{j}\left(\xi, z_{j}, \omega\right),
\end{array}\right\},
$$

where $\mathbf{E}^{j}(\hbar)=\left[\begin{array}{cc}e^{-\gamma_{1}^{j} \hbar} & 0 \\ 0 & e^{-\gamma_{2}^{j} \hbar}\end{array}\right], j=1,2, N$.

The continuity condition of the layered soil in the frequency wave-number domain is given by

$$
\Psi^{j}\left(\xi, z_{j}, \omega\right)=\Psi^{j+1}\left(\xi, z_{j}, \omega\right), \quad j=1,2, \ldots, N-1 .
$$

Substituting Equation (14) into Equation (16) gives a new form as follows:

$$
\left[\begin{array}{cc}
-\mathbf{D}_{\mathbf{d}}^{j+1} & \mathbf{D}_{\mathbf{u}}^{j} \\
-\mathbf{S}_{\mathbf{d}}^{j+1} & \mathbf{S}_{\mathbf{u}}^{j}
\end{array}\right]\left[\begin{array}{c}
\mathbf{W}_{\mathbf{d}}^{j+1}\left(z_{j}\right) \\
\mathbf{W}_{\mathbf{u}}^{j}\left(z_{j}\right)
\end{array}\right]=\left[\begin{array}{cc}
-\mathbf{D}_{\mathbf{d}}^{j} & \mathbf{D}_{\mathbf{u}}^{j+1} \\
-\mathbf{S}_{\mathbf{d}}^{j} & \mathbf{S}_{\mathbf{u}}^{j+1}
\end{array}\right]\left[\begin{array}{c}
\mathbf{W}_{\mathbf{d}}^{j}\left(z_{j}\right) \\
\mathbf{W}_{\mathbf{u}}^{j+1}\left(z_{j}\right)
\end{array}\right]
$$

Matrix inverse operation on Equation (17) can be obtained as follows:

$$
\left[\begin{array}{c}
\mathbf{W}_{\mathbf{d}}^{j+1}\left(z_{j}\right) \\
\mathbf{W}_{\mathbf{u}}^{j}\left(z_{j}\right)
\end{array}\right]=\left[\begin{array}{ll}
\mathbf{T}_{\mathbf{d}}^{j}(\xi, \omega) & \mathbf{R}_{\mathbf{u}}^{j}(\xi, \omega) \\
\mathbf{R}_{\mathbf{d}}^{j}(\xi, \omega) & \mathbf{T}_{\mathbf{u}}^{j}(\xi, \omega)
\end{array}\right]\left[\begin{array}{c}
\mathbf{W}_{\mathbf{d}}^{j}\left(z_{j}\right) \\
\mathbf{W}_{\mathbf{u}}^{j+1}\left(z_{j}\right)
\end{array}\right],
$$

where $\mathbf{R}_{\mathbf{u}}^{j}$ and $\mathbf{R}_{\mathbf{d}}^{j}$ are represented by the reflection matrix of up going and down going wave, respectively; $\mathbf{T}_{\mathbf{d}}^{j}$ and $\mathbf{T}_{\mathbf{u}}^{j}$ are represented by the transmission matrix of up going and down going wave, respectively. They can be expressed as follows:

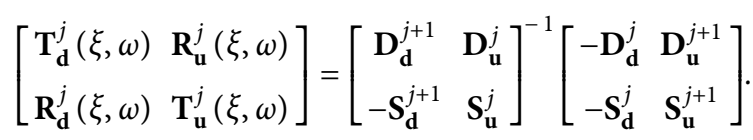

The down going wave vector of each layer can be represented by the wave vector of the corresponding upper layer, and the up going wave vector can be expressed by the down going wave vector of this layer according to Equations (5a) and (5b). Therefore, the wave vector of each layer under the tunnel structure can be denoted by the down going wave vector $\mathbf{W}_{\mathbf{d}}^{L}\left(\xi, z_{L}, \omega\right)$ of the layer subjected to a moving load, recursively:

$$
\left.\begin{array}{l}
\mathbf{W}_{\mathbf{d}}^{j}\left(\xi, z_{j-1}, \omega\right)=\mathbf{T}_{\mathbf{d e}}^{\mathbf{g}(j-1)} \mathbf{T}_{\mathbf{d e}}^{\mathbf{g}(j-2)} \cdots \mathbf{T}_{\mathbf{d e}}^{\mathbf{g} 2} \mathbf{T}_{\mathbf{d}}^{g 1} \mathbf{W}_{\mathbf{d}}^{L}\left(\xi, z_{L}, \omega\right), \\
\mathbf{W}_{\mathbf{u}}^{j}\left(\xi, z_{j}, \omega\right)=\mathbf{R}_{\mathbf{d}}^{\mathbf{g} j} \mathbf{W}_{\mathbf{d}}^{j}\left(\xi, z_{j}, \omega\right), \\
\mathbf{R}_{\mathbf{d}}^{g j}=\mathbf{R}_{\mathbf{d}}^{j}+\mathbf{T}_{\mathbf{u e}}^{j} \mathbf{R}_{\mathbf{d e}}^{j+1} \mathbf{T}_{\mathbf{d}}^{\mathbf{g} j}, \\
\mathbf{T}_{\mathbf{d e}}^{g j}=\left(I-\mathbf{R}_{\mathbf{u e}}^{j} \mathbf{R}_{\mathbf{d e}}^{\mathbf{g} j+1}\right)^{-1} \mathbf{T}_{\mathbf{d e}}^{j},
\end{array}\right\}
$$

where $\left[\begin{array}{cc}\mathbf{T}_{\mathbf{d e}}^{j} & \mathbf{R}_{\mathbf{u e}}^{j} \\ \mathbf{R}_{\mathbf{d e}}^{j} & \mathbf{T}_{\mathbf{u e}}^{j}\end{array}\right]=\left[\begin{array}{cc}\mathbf{T}_{\mathbf{d}}^{j} & \mathbf{R}_{\mathbf{u}}^{j} \\ \mathbf{R}_{\mathbf{d}}^{j} & \mathbf{T}_{\mathbf{u}}^{j}\end{array}\right]\left[\begin{array}{cc}\mathbf{E}^{j}\left(z_{j}\right) & 0 \\ 0 & \mathbf{E}^{j+1}\left(z_{j+1}\right)\end{array}\right]$ and $\left[\begin{array}{cc}\mathbf{T}_{\mathbf{d e}}^{\mathbf{g} j} & \mathbf{R}_{\mathbf{u e}}^{\mathbf{g} j} \\ \mathbf{R}_{\mathbf{d e}}^{\mathbf{g} j} & \mathbf{T}_{\mathbf{u e}}^{\mathbf{g} j}\end{array}\right]=\left[\begin{array}{cc}\mathbf{T}_{\mathbf{d}}^{\mathbf{g} j} & \mathbf{R}_{\mathbf{u}}^{\mathbf{g} j} \\ \mathbf{R}_{\mathbf{d}}^{\mathbf{g} j} & \mathbf{T}_{\mathbf{u}}^{\mathbf{g} j}\end{array}\right]\left[\begin{array}{cc}\mathbf{E}^{j}\left(z_{j}\right) & 0 \\ 0 & \mathbf{E}^{j+1}\left(z_{j+1}\right)\end{array}\right], \quad j=L+1$, $L+2, \ldots, N$.

In the same way, the wave vector of each layer above the tunnel structure can also be denoted by the up going wave vector $\mathbf{W}_{\mathbf{u}}^{L}\left(\xi, z_{L-1}, \omega\right)$ of the layer subjected to a moving load:

$$
\left.\begin{array}{l}
\mathbf{W}_{\mathbf{u}}^{j}\left(\xi, z_{j}, \omega\right)=\mathbf{T}_{u \mathbf{e}}^{\mathbf{g} j} \mathbf{T}_{u \mathbf{e}}^{\mathbf{g}(j+1)} \cdots \mathbf{T}_{\mathbf{d e}}^{\mathbf{g}(L-2)} \mathbf{T}_{u}^{\mathbf{g}(L-1)} \mathbf{W}_{u}^{L}\left(\xi, z_{L-1}, \omega\right), \\
\mathbf{W}_{\mathbf{d}}^{j}\left(\xi, z_{j-1}, \omega\right)=\mathbf{R}_{u \mathbf{e}}^{\mathbf{g}(j-1)} \mathbf{W}_{u}^{j}\left(\xi, z_{j}, \omega\right), \\
\mathbf{R}_{u}^{\mathbf{g} j}=\mathbf{R}_{u}^{j}+\mathbf{R}_{d \mathbf{e}}^{j} \mathbf{R}_{u \mathbf{e}}^{j-1} \mathbf{T}_{u}^{\mathbf{g} j}, \\
\mathbf{T}_{u}^{\mathbf{g} j}=\left(I-\mathbf{R}_{d \mathbf{e}}^{j} \mathbf{R}_{u \mathbf{e}}^{\mathbf{g}(j-1)}\right)^{-1} \mathbf{T}_{\mathbf{u}}^{j},
\end{array}\right\}
$$

where $j=1,2, \ldots, L-1$.

Due to the stress jump appeared in the $L$ th-layer soil where the tunnel structure is located, the up going and down going wave vectors $\left\{\mathbf{W}_{d}^{L}(\xi, z, \omega), \mathbf{W}_{u}^{L}(\xi, z, \omega)\right\}$ of the layer can be expressed as follows:

$$
\begin{aligned}
& a^{L}(\xi, \omega)=a^{l 1}(\xi, \omega)+H(s-z) a^{l 2}(\xi, \omega), \\
& c^{L}(\xi, \omega)=c^{l 1}(\xi, \omega)+H(s-z) c^{l 2}(\xi, \omega), \\
& b^{L}(\xi, \omega)=b^{l 1}(\xi, \omega)+H(z-s) b^{l 2}(\xi, \omega) . \\
& d^{L}(\xi, \omega)=d^{l 1}(\xi, \omega)+H(z-s) d^{l 2}(\xi, \omega) .
\end{aligned}
$$

The wave vectors of the $L$ th layer soil can be obtained by means of the TRM method:

$$
\left.\begin{array}{l}
\left\{\begin{array}{ll}
b^{l 1} & d^{l 1}
\end{array}\right\}^{T}=\stackrel{g}{R_{u}} E(h)\left\{\begin{array}{ll}
a^{l 1} & c^{l 1}
\end{array}\right\}^{T}, \\
\left\{\begin{array}{ll}
a^{l 2} & c^{l 2}
\end{array}\right\}^{T}=\stackrel{g}{R}_{d} E(H)\left\{\begin{array}{ll}
b^{l 2} & d^{l 2}
\end{array}\right\}^{T} .
\end{array}\right\},
$$

Considering the continuous boundary conditions of the displacement at the soil-tunnel structure interface, the general solutions of Equations (4a), (4b), and (14) can be written as follows:

$$
\begin{aligned}
& D_{d}^{l 1} E(h)\left\{\begin{array}{c}
b^{l 1} \\
d^{l 1}
\end{array}\right\}+D_{u}^{l 1}\left\{\begin{array}{c}
a^{l 1} \\
c^{l 1}
\end{array}\right\}=\left\{\begin{array}{c}
0 \\
w_{b}
\end{array}\right\}, \\
& D_{d}^{l 2}\left\{\begin{array}{l}
b^{l 2} \\
d^{l 2}
\end{array}\right\}+D_{u}^{l 2} E(H)\left\{\begin{array}{c}
a^{l 2} \\
c^{l 2}
\end{array}\right\}=\left\{\begin{array}{c}
0 \\
w_{b}
\end{array}\right\} .
\end{aligned}
$$

Taking into account the stress balance boundary conditions of the displacement at the soil-tunnel structure interface, one may write the equation as 


$$
\begin{gathered}
a\left[S_{d \sigma z}^{l 2} E(H)\left\{\begin{array}{l}
b^{l 2} \\
d^{l 2}
\end{array}\right\}+S_{u \sigma z}^{l 2}\left\{\begin{array}{c}
a^{l 2} \\
c^{l 2}
\end{array}\right\}\right] \\
-a\left[S_{d \sigma z}^{l 1} E(h)\left\{\begin{array}{c}
b^{l 1} \\
d^{l 1}
\end{array}\right\}+S_{u \sigma z}^{l 1}\left\{\begin{array}{c}
a^{l 1} \\
c^{l 1}
\end{array}\right\}\right]=q,
\end{gathered}
$$

where $q$ denotes the reaction force of soil foundation, and $S_{d \sigma z}^{l 1}, S_{u \sigma z}^{l 1}, S_{d \sigma z}^{l 2}$, and $S_{u \sigma z}^{l 2}$ are determined by Equation (14).

The equivalent stiffness matrix between reaction force $q$ of the layered soil foundation and displacement $w_{b}$ of the viscoelastic Euler beam can be obtained in accordance with Equation (23). Substitution of the equivalent stiffness matrix into Equation (14) yields the following expression for solutions of the surface ground vibration.

As far as the tunnel structure due to a moving load is concerned, the wave vectors of viscoelastic beam layer can be solved by Equation (22) and the others by Equations (19) and (20). Finally, the displacement and stress with respect to the frequency wave-number solution can be derived by Equation (14). Universally, all variables in the frequency wavenumber domain are expressed by $\widetilde{\Omega}$ as follows:

$$
\widetilde{\bar{\Omega}}(\xi, z, \omega)=\tilde{\bar{\Omega}}^{*}(\xi, z, \omega) F_{z} \delta(\omega+\xi c)
$$

\section{Numerical Results and Discussions}

Applying the double inverse Fourier transforms to Equation (16), one obtains the general solutions inthe time-space domain. Moreover, the above transforms can be simplified in accordance with the function of Dirac- $\delta$ :

$$
\Omega(x, z, t)=\left(\frac{1}{2 \pi}\right)^{2} F_{z} \int_{-\infty}^{+\infty} \tilde{\bar{\Omega}}(\xi, z,-\xi c) e^{i \xi\left(x-v_{c} t\right)} \mathrm{d} \xi .
$$

It is difficult to obtain a closed-form solution of the Fourier inverse transform due to the complexity of the integrand. This article uses the FFT method to complete the Fourier inverse transform. Therefore, the FFT method is adopted to complete the inverse transforms in this paper. In addition, the existence of soil viscosity will prevent branch points and singularities on the integration path of the horizontal wave-number $\xi$ caused by the application of the viscoelastic model. The scale of discrete sample points for wave-number $\xi$ is $N=4096$ [6], the space interval is $\Delta x=0.1$, and the calculation space is $L_{x}=N \Delta x$.

In the following sections, four types of surface ground vibration due to a moving load for a three-layered soilviscoelastic Euler beam system will be considered. A moving point load with an amplitude $F_{z}$ acts on the viscoelastic beam in the layered soil at a constant velocity $v_{c}$ along the positive direction of the $x$-axis. Each layer of the soil foundation with their thickness of $h_{1}=h_{2}=3.5 \mathrm{~m}$ and $h_{3}=15.0 \mathrm{~m}$ has the same density $\rho=1.7 \times 10^{3} \mathrm{~kg} / \mathrm{m}^{3}$ and the same Poisson's ratio $v=0.3$, respectively. For each layer, three different values of the shear modulus $\mu_{s}^{(i)}=E_{s}^{(i)} /\left(2.0 *\left(1+v_{s}\right)\right)(i=1,2,3)$, respectively, are considered, where $v_{s}=\sqrt{\mu_{s}^{(2)} / \rho_{s}}$ [6]. In this paper, a standard solid model is used in the viscoelastic beam system with the parameters of $E_{p}^{(1)}$ and $E_{p}^{(2)}$.

4.1. Example 1. For a homogeneous soil foundation, the material parameters for the layered soil are given as follows: $E_{s}^{(i)}=2.0 \times 10^{9} \mathrm{~Pa}, E^{*}=3.0 \times 10^{4} \mathrm{~Pa}, E_{p}^{(1)}=E_{p}^{(2)}=2.0 \times 10^{9} \mathrm{~Pa}$, and $\eta=0.0$. Figure 2 shows that the variation of vertical and horizontal displacements located at the point $A(0.0,0.0 \mathrm{~m})$ when the load is moving with an amplitude $F_{z}=1.0 \times 10^{4} \mathrm{~N} / \mathrm{m}$ and a velocity $v_{c}=30.0 \mathrm{~m} / \mathrm{s}$ with time $t$. In Figure $2(\mathrm{a})$, the maximum and minimum values of present solution are $1.5 \mathrm{~mm}$ and $-1.52 \mathrm{~mm}$, respectively, which are very close to the results of Metrikine et al. [6]. The curve in Figure 2(b) is funnel-shaped, and the minimum value is $4.8 \mathrm{~mm}$. This result is slightly less than that of Metrikine et al. [6]. It can be seen from Figure 2 that the current solution is very close to the solution in the literature, and the maximum difference is not more than $0.2 \mathrm{~mm}$. Therefore, it can be considered that the results in present solution are consistent with those in [6].

4.2. Example 2. The section aimed to study the effect of elastic modulus and viscosity coefficient on the surface ground vibration. The material parameters for the layered soil are given as follows: $E_{p}^{(1)}=E_{p}^{(2)}=1.0 \times 10^{9} \mathrm{~Pa}$, $2.0 \times 10^{9} \mathrm{~Pa}, 5.0 \times 10^{9} \mathrm{~Pa}$, and $\eta=3.0 \times 10^{5} \mathrm{~Pa}$. The values of other calculation parameters are the same as in example 1 . The moving point load acts on the viscoelastic beam in the layered soil along the $x$-axis at the velocity $v_{c}=0.5 v_{s}$.

Figure 3 shows the variation of the shear modulus with the calculation frequency of viscoelastic beam with respect to the different elastic parameters. As shown in Figure 3, the shear modulus is a function of frequency and increases as the elastic modulus of the viscoelastic beam.

In Figure 4, the variation of vertical and horizontal displacements located at the observation point $A(0.0,0.0 \mathrm{~m})$ with time $t$ are plotted as solid lines following the different elasticity parameters, respectively.

As shown in Figure 4, the surface ground vibration of layered soil decreases with the increase of the shear modulus, and the attenuation of vibration becomes slower. It can be seen that the greater the rigidity of the track beam structure, the greater the energy dissipated as an energy absorbing layer, and the smaller the energy transmitted in the free field of the foundation caused by the load acting on the surrounding soil through the beam.

4.3. Example 3. In order to investigate the effect of different viscosity coefficients with respect to the viscoelastic beam, a series of the viscosity coefficients $\eta=3.0 \times 10^{5} \mathrm{~Pa}$, $3.0 \times 10^{7} \mathrm{~Pa}$, and $3.0 \times 10^{9} \mathrm{~Pa}$ are utilized to simulate. The elastic parameters for the layered soil are given as follows: $E_{p}^{(1)}=E_{p}^{(2)}=2.0 \times 10^{9} \mathrm{~Pa}$, and the values of other calculation parameters are the same as those in example 1. 


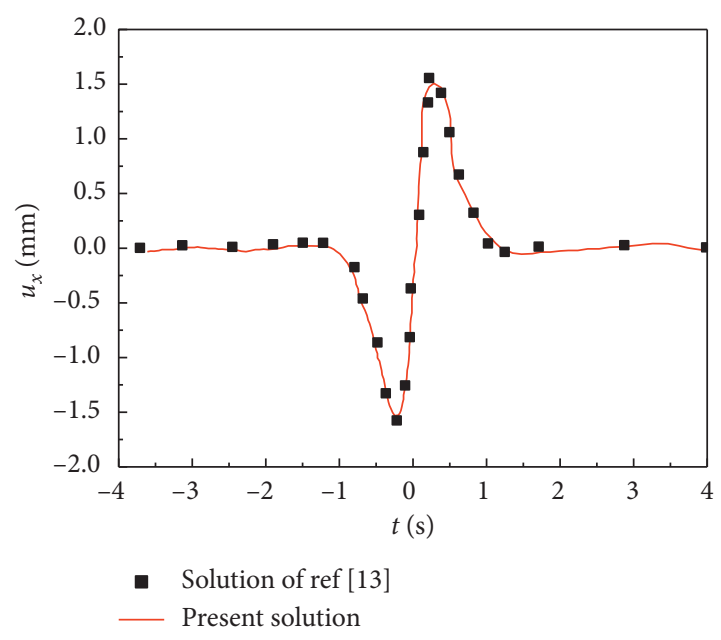

(a)

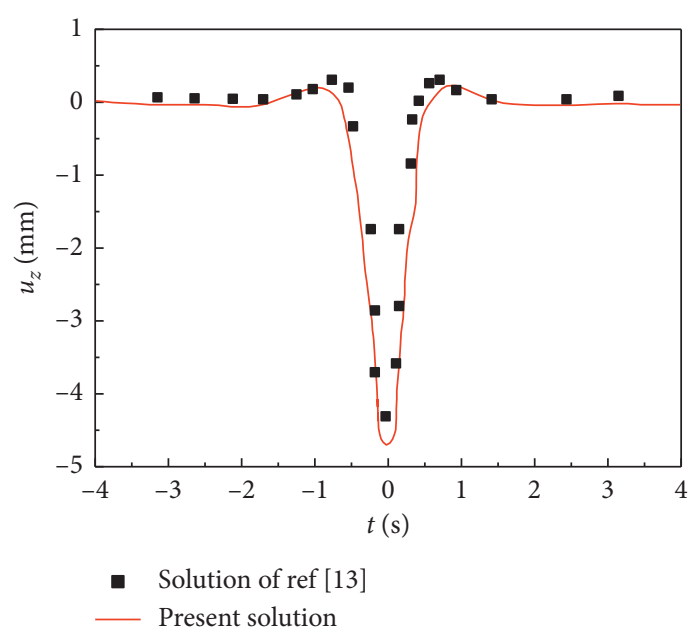

(b)

Figure 2: Comparison with reference results: (a) horizontal displacement; (b) vertical displacement.

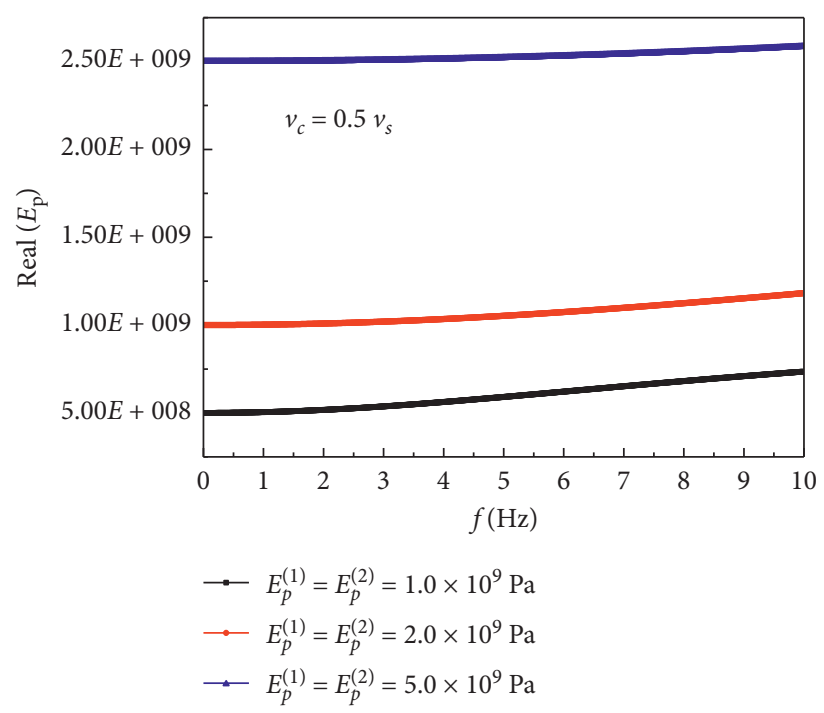

FIgURE 3: Variation of shear modulus of viscoelastic beam with frequency under different elastic parameters.

The moving point load acts on the viscoelastic beam in the layered soil along the $x$-axis at the velocity $v_{c}=0.5 v_{s}$.

Figure 5 shows the variation of the shear modulus with the calculation frequency of viscoelastic beam. As shown in Figure 5 , when the viscosity coefficient of the viscoelastic beam $\eta$ is unequal to zero, the elastic modulus of the beam is a complex modulus, and it increases with the increase of the calculation frequency. However, when the viscosity coefficient increases to a certain value $\left(\eta=3.0 \times 10^{9} \mathrm{~Pa}\right.$, e.g. $)$, the viscoelastic Euler beam behaves as plastic, and its elastic modulus will not change with the calculation frequency after a certain frequency. Thus, the standard solid model of viscoelastic beam can describe the creep and relaxation of materials properly.
Figure 6 shows that the variation of vertical and horizontal displacements located at the observation point $A(0.0$, $0.0 \mathrm{~m}$ ) with time $t$ under different elastic parameters, respectively. As shown in Figure 6, as the viscosity coefficient of the beam increases, the vibration amplitude of the soil surface will decrease, but the impact is limited.

4.4. Example 4. The effect of the differentiation of layered soil on the surface vibration subjected to a moving load will be investigated in this section. There are three cases with respect to the complex Lame constant of layered soil: CASE (A) $\mu^{(1)}: \mu^{(2)}: \mu^{(3)}=1: 1: 1$, CASE (B) $\mu^{(1)}: \mu^{(2)}: \mu^{(3)}=0.1: 1: 1$, 


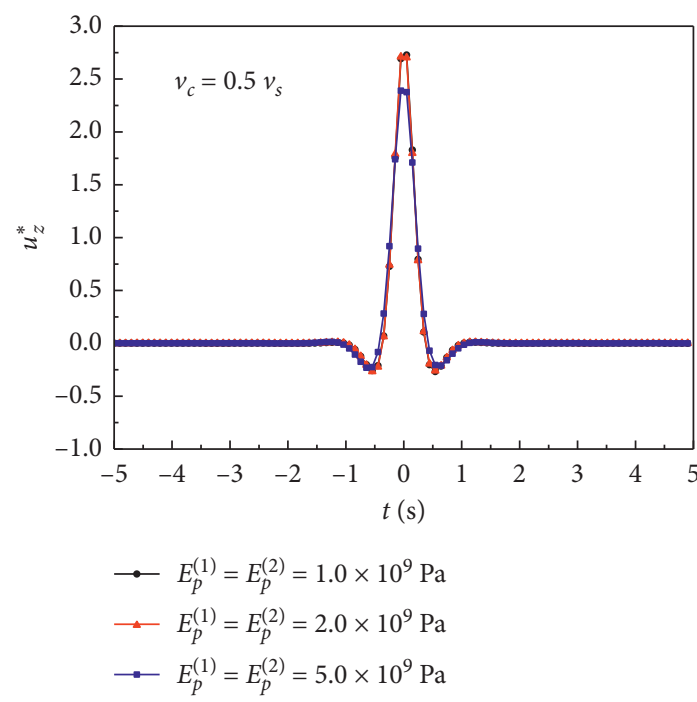

(a)

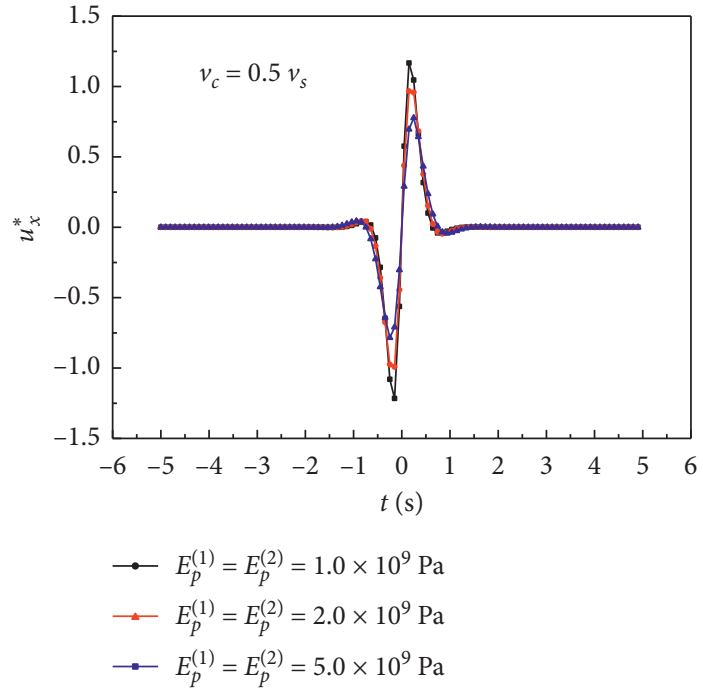

(b)

Figure 4: Variation of the observation point $A(0.0,0.0 \mathrm{~m})$ displacement with time $t$ under different elastic parameters: (a) vertical displacement; (b) horizontal displacement.

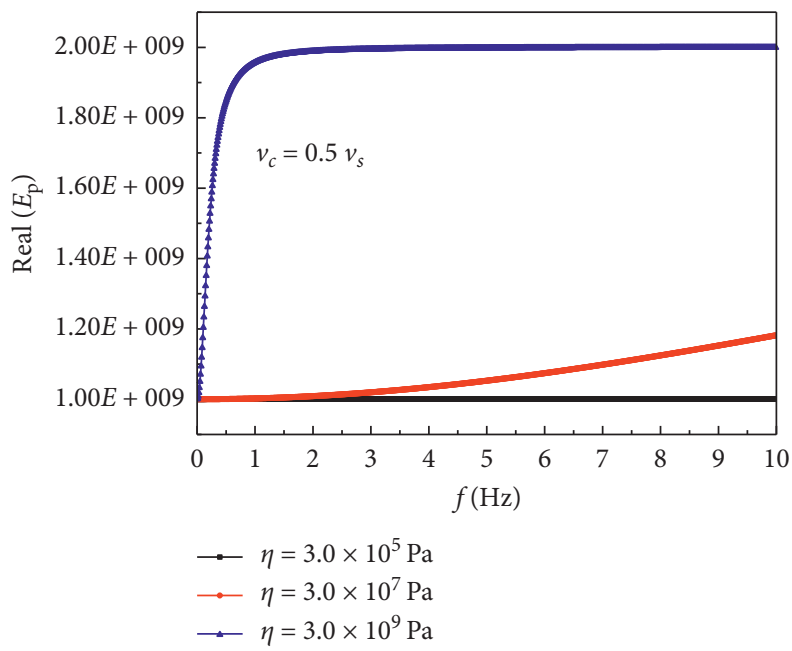

FIGURE 5: Variation of the observation point $A(0.0,0.0 \mathrm{~m})$ displacement with time $t$ under different elastic parameters.

and CASE (C) $\mu^{(1)}: \mu^{(2)}: \mu^{(3)}=1: 1: 10$, respectively. The material parameters for the layered soil are given as follows: $E_{s}^{(2)}=3.0 \times 10^{7} \mathrm{~Pa}, \quad E_{p}^{(1)}=E_{p}^{(2)}=2.0 \times 10^{9} \mathrm{~Pa}, \quad$ and $\eta=3.0 \times 10^{7} \mathrm{~Pa}$, and the velocity of moving point load $v_{c}=0.2 v_{s}, v_{c}=0.5 v_{s}$, and $v_{c}=1.2 v_{s}$ respectively. The values of other calculation parameters are the same as those in example 1.

The variation of vertical and horizontal displacements spectrum with frequency located at the observation point $A$ $(0.0,0.0 \mathrm{~m})$ under different layered soil is shown in Figures 7 and 8 , respectively.
From Figures 7 and 8, one can see that when the load velocity is rather low $\left(v_{c}=0.2 v_{s}\right.$, e.g.), the vertical and horizontal displacements spectrum of the observation point are mainly distributed in the lower frequency range, whereas the load velocity exceeds the shear wave velocity of the soil, and the distribution of the vertical and horizontal displacements spectrum of the observation point increases almost 2 times than before. In addition, for the layered soil with weak interlayer, the values of vertical and horizontal displacements spectrum with respect to the observation point will increase and with the increase of the load velocity. 


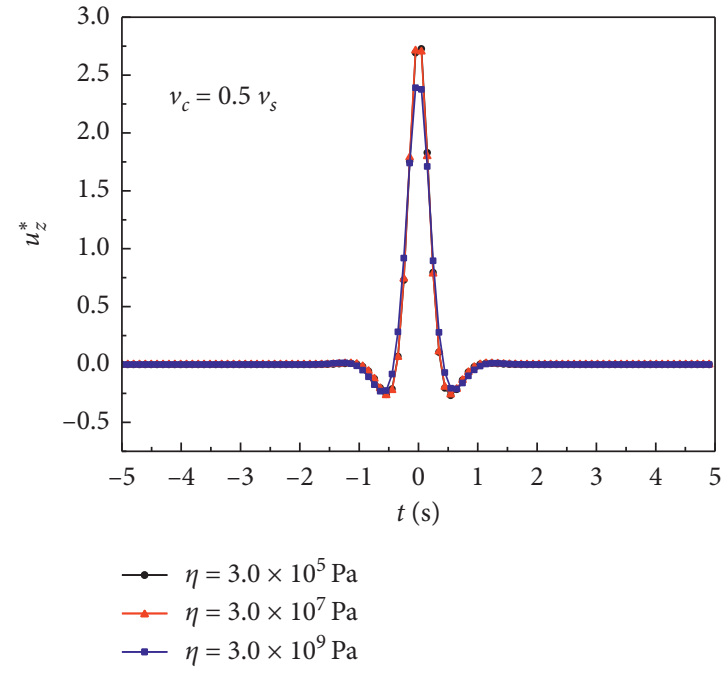

(a)

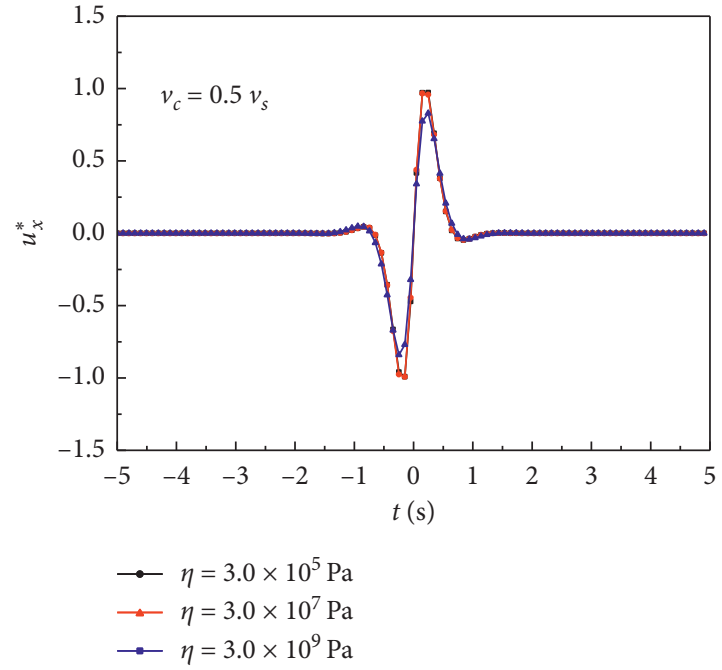

(b)

FIgURE 6: Variation of the observation point $A(0.0,0.0 \mathrm{~m})$ displacement with time $t$ under different elastic parameters: (a) vertical displacement; (b) horizontal displacement.

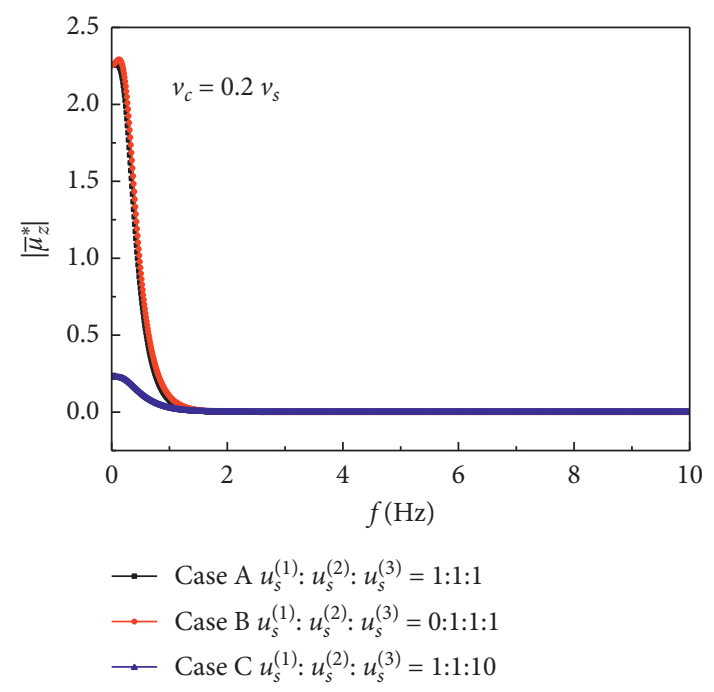

(a)

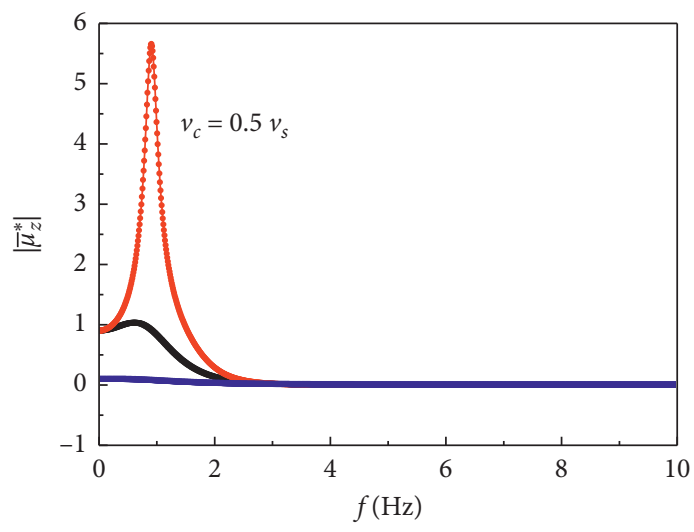

$\rightarrow$ Case A $u_{s}^{(1)}: u_{s}^{(2)}: u_{s}^{(3)}=1: 1: 1$
$\rightarrow$ Case B $u_{s}^{(1)}: u_{s}^{(2)}: u_{s}^{(3)}=0: 1: 1: 1$
$\rightarrow$ Case C $u_{s}^{(1)}: u_{s}^{(2)}: u_{s}^{(3)}=1: 1: 10$

(b)

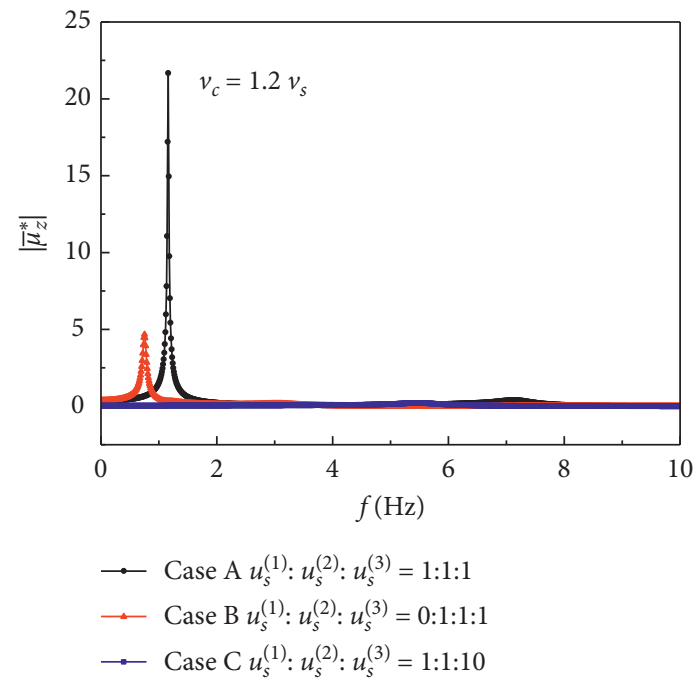

(c)

FIGURE 7: Variation of the observation point $A(0.0,0.0 \mathrm{~m})$ vertical displacement spectrum with frequency under different layered soil: (a) $v_{c}=0.2 v_{s}$, (b) $v_{c}=0.5 v_{s}$, (c) $v_{c}=1.2 v_{s}$. 


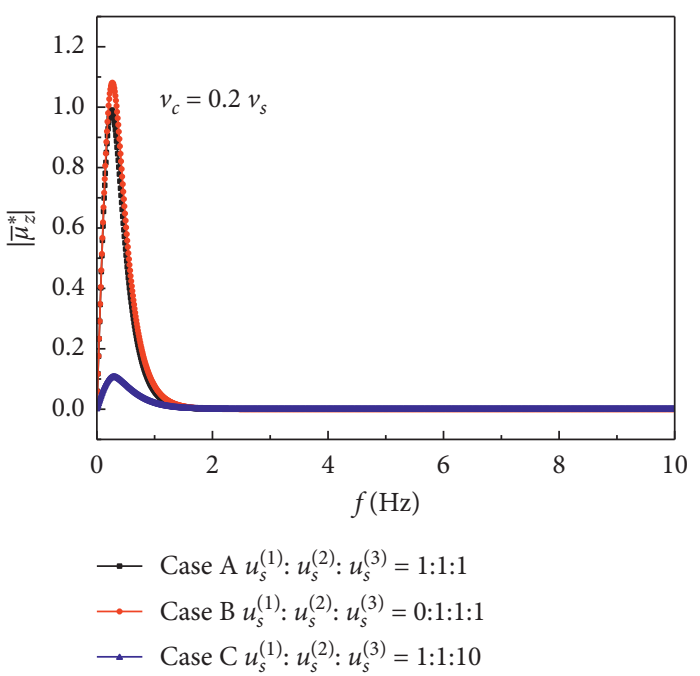

(a)

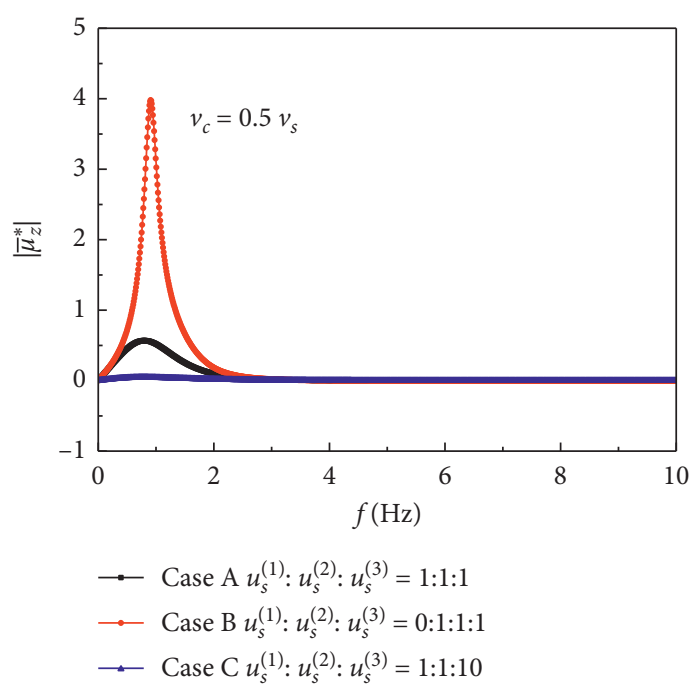

(b)

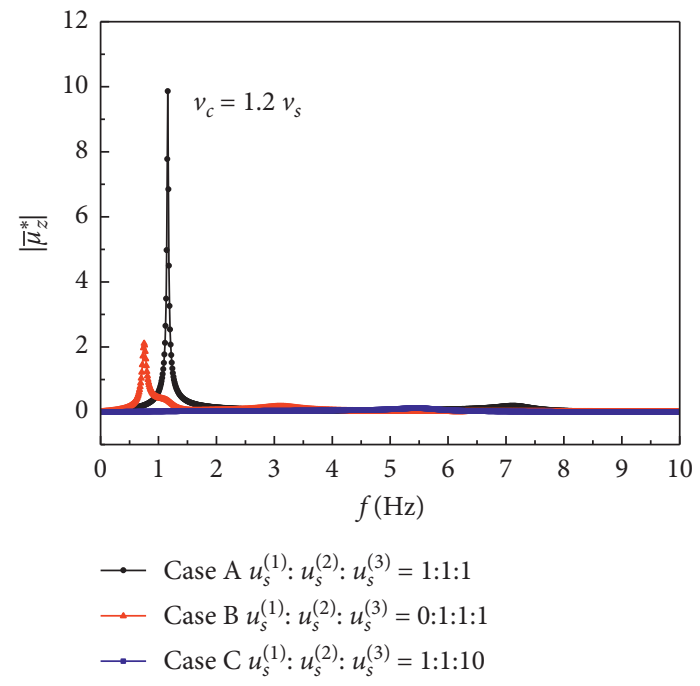

(c)

FiguRE 8: Variation of the observation point $A(0.0,0.0 \mathrm{~m})$ horizontal displacement spectrum with frequency under different layered soil: (a) $v_{c}=0.2 v_{s}$, (b) $v_{c}=0.5 v_{s}$, (c) $v_{c}=1.2 v_{s}$.

The peak displacement spectrum of the observation point appears at a smaller frequency position at the same time, and the approximate number of the peak displacement spectrum also increases. On the contrary, for the layered soil with hard interlayer, the above conclusion will be just the opposite.

The variation of vertical and horizontal displacements spectrum located at the observation point $A(0.0,0.0 \mathrm{~m})$ with time $t$ under different layered soil is plotted in Figures 9 and 10 , respectively. One can see from the figures that when the load velocity is rather low (e.g., $v_{c}=0.2 v_{s}$ ), the dynamic response in the time domain of the vertical and horizontal displacements located at the observation point is symmetrically distributed on both sides of the loading arrival time. As the load velocity increases, the displacement amplitude of the observation point increases and the vibration duration of the soil surface is longer, then its symmetry with respect to the moment of loading arrival will be disappeared accompanied by the phenomenon of shock wave. Figures 9 and 10 obviously show that the wave pattern for $t<0$ has a higher frequency and smaller amplitude than that for $t>0$. The difference in frequencies is because of the Doppler effect, implying that an observed wave possesses a higher frequency when the load moves towards the observation point $(t<0)$, and, on the other hand, the wave frequency becomes smaller when the distance between the load and the observation point grows $(t>0)$.

Furthermore, the vertical and horizontal displacements of the observation point provided that $v_{c}=0.2 v_{s}$ are the 


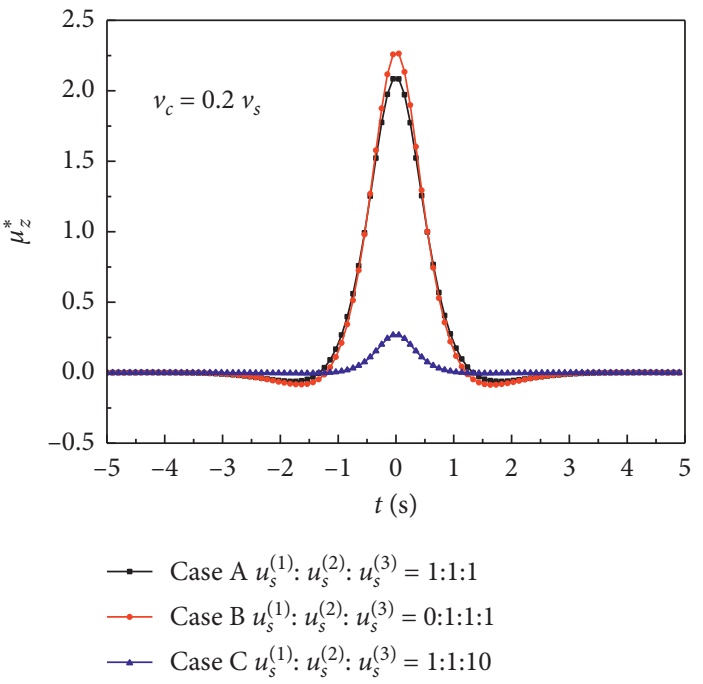

(a)

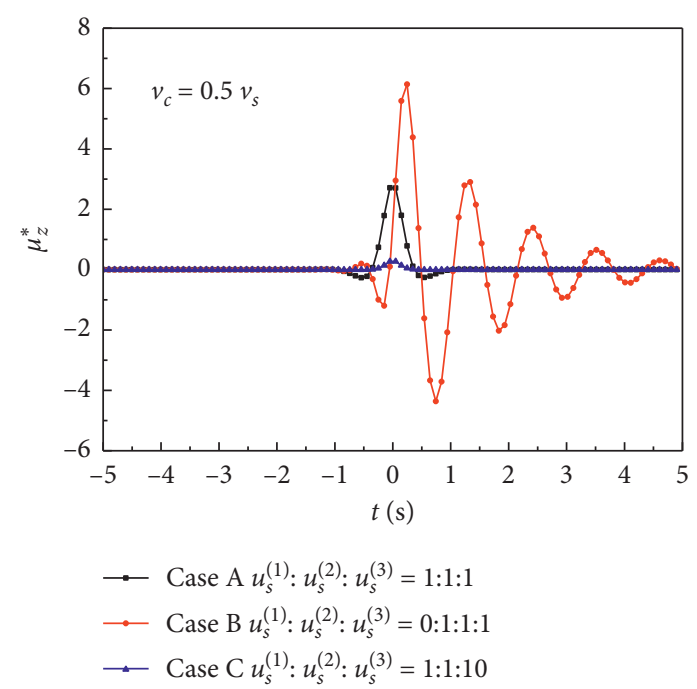

(b)

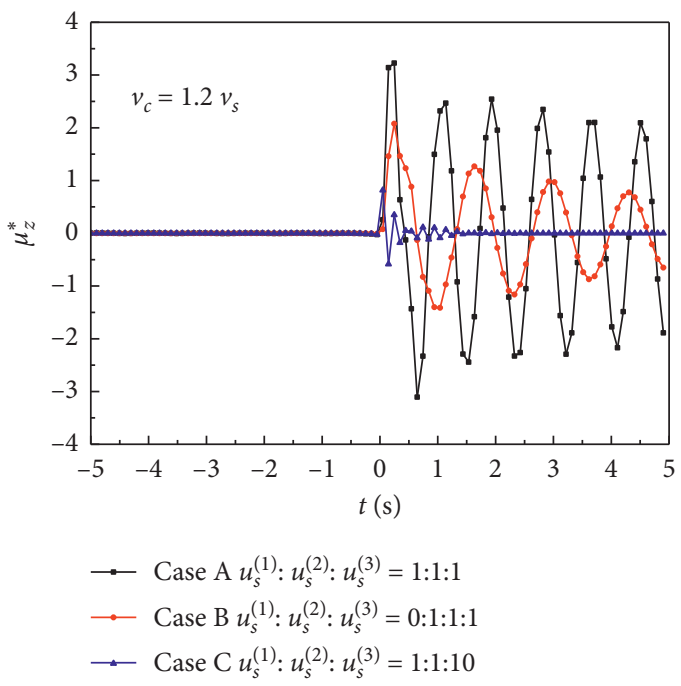

(c)

FiguRE 9: Variation of the observation points $A(0.0,0.0 \mathrm{~m})$ vertical displacement spectrum with time $t$ under different layered soil: (a) $v_{c}=0.2 v_{s}$, (b) $v_{c}=0.5 v_{s}$, (c) $v_{c}=1.2 v_{s}$.

largest in CASE (B), followed by CASE (A) and CASE (C), implying that surface vibration of layered soil with weak interlayer is greater than that with stiffer layer in the case of lower load velocity. Nevertheless, the vertical and horizontal displacements of the observation point with weak interlayer in the layered soil can reach the maximum value in the case of $v_{c}=0.5 v_{s}$, and there is definite fluctuation. For a homogeneous soil foundation, the vertical and horizontal displacement amplitudes of the observation point are the largest in the case of $v_{c}=1.2 v_{s}$. The existence of weak interlayer in the soil foundation makes the vibration attenuation slower. 

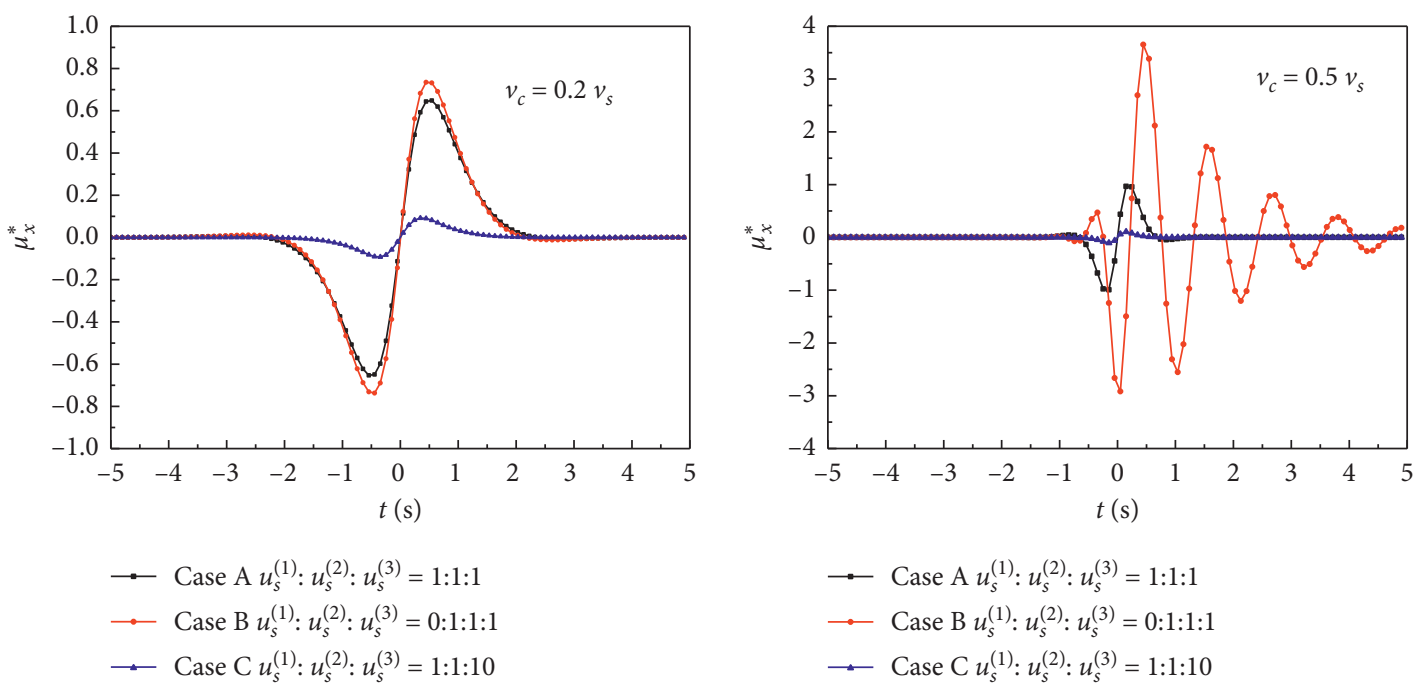

(a)

(b)

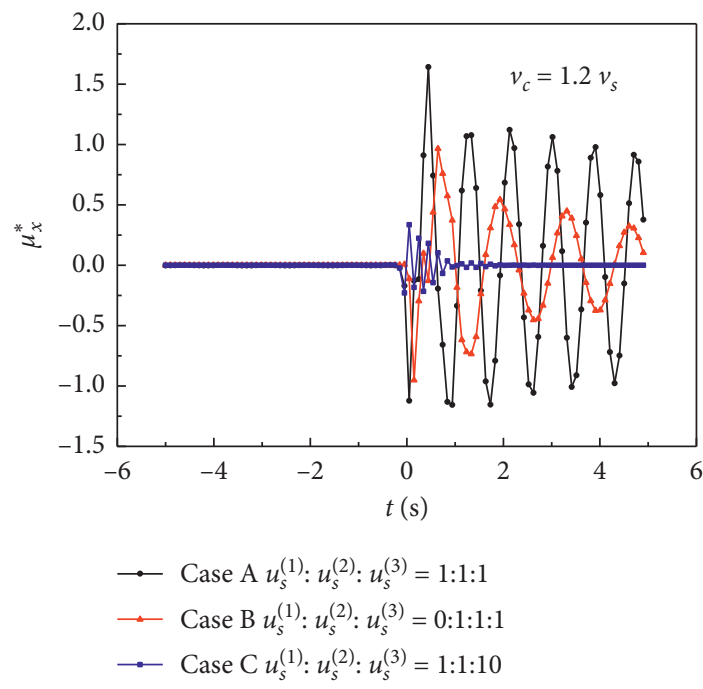

(c)

Figure 10: Variation of the observation points $A(0.0,0.0 \mathrm{~m})$ horizontal displacement spectrum with time $t$ under different layered soil: (a) $v_{c}=0.2 v_{s}$, (b) $v_{c}=0.5 v_{s}$, (c) $v_{c}=1.2 v_{s}$.

\section{Conclusion}

In this paper, considering the viscoelasticity of the concrete material of the subway tunnel structure and the layered soil foundation, using the standard linear elastic solid model and the TRM method, the characteristics of the vibration of the ground surface caused by the different load velocity in the layered soil foundation are analyzed. Based on the numerical simulations performed in this study, following conclusions can be drawn:

(1) As the viscosity coefficient of the viscoelastic beam increases, the vibration amplitude of the soil surface will decrease, but the impact is limited. Therefore, the creep and relaxation of the material can be described by the standard solid model for viscoelastic beams, which reflect the working conditions of the concrete structure for subway tunnel properly.
(2) As the elastic modulus of the viscoelastic element increases, the shear modulus of the viscoelastic beam increases, the vibration of the layered soil surface will decrease, and the vibration attenuation will slow down. It can be seen that the greater the rigidity of the track beam structure, the greater the energy dissipated as an energy absorbing layer, and the smaller the energy transmitted in the free field of the foundation caused by the load acting on the surrounding soil through the beam.

(3) When the load velocity is rather low, the displacement spectra of vibrations are mainly distributed in the lower frequency range, whereas the load velocity exceeds the shear wave velocity of the soil, and the spectrum is approximately twice as wide.

(4) For the layered soil with weak interlayer, the displacements spectrum will increase with the increase 
of the load velocity. The peak displacement spectrum appears at a smaller frequency position at the same time, and the approximate number of the peak displacement spectrum also increases. On the contrary, for the layered soil with hard interlayer, the above concludes will be just the opposite.

(5) The vertical and horizontal displacements of the observation point with weak interlayer in the layered soil can reach the maximum value in the case of $v_{c}=0.5 v_{s}$, and there is definite fluctuation. For a homogeneous soil foundation, the amplitude spectrum of vibration will peak while the load velocity exceeds the shear wave velocity of the soil. The existence of weak interlayer in the soil foundation makes the vibration attenuation slower.

\section{Data Availability}

The data used to support the findings of this study are included within the article.

\section{Conflicts of Interest}

The authors declare that they have no conflicts of interest.

\section{Acknowledgments}

This work was financially supported by the National Science Foundation of China (Nos. 51569016 and 5206080257), the Jiangxi Province Department of Education Science and technology research project (Nos. GJJ12629, GJJ171008, and GJJ11253), and Key projects of Natural Science Foundation of Jiangxi Province (Nos. 20114BAB206012 and 20181BAB216028).

\section{References}

[1] N. Bendenia, M. Zidour, A. A. Bousahla et al., "Deflections, stresses and free vibration studies of FG-CNT reinforced sandwich plates resting on pasternak elastic foundation," Computers and Concrete, vol. 26, no. 3, pp. 213-226, 2020.

[2] O. Allam, K. Draiche, A. A. Bousahla et al., "A generalized 4unknown refined theory for bending and free vibration analysis of laminated composite and sandwich plates and shells," Computers and Concrete, vol. 26, no. 2, pp. 185-201, 2020.

[3] A. Menasria, A. Kaci, A. A. Bousahla et al., "A four-unknown refined plate theory for dynamic analysis of FG-sandwich plates under various boundary conditions," Steel and Composite Structures, vol. 36, no. 3, pp. 355-367, 2020.

[4] A. Zine, A. A. Bousahla, F. Bourada et al., "Bending analysis of functionally graded porous plates via a refined shear deformation theory," Computers and Concrete, vol. 26, no. 1, pp. $63-74,2020$.

[5] M. Rabhi, K. H. Benrahou, A. Kaci et al., "A new innovative 3unknowns HSDT for buckling and free vibration of exponentially graded sandwich plates resting on elastic foundations under various boundary conditions," Geomechanics and Engineering, vol. 22, no. 2, pp. 119-132, 2020.

[6] Y. B. Yang and H. H. Hung, "Soil vibrations caused by underground moving trains," Journal of Geotechnical and
Geoenvironmental Engineering, vol. 134, no. 11, pp. 16331644, 2008.

[7] X. C. Bian, W. F. Jin, and H. G. Jiang, "Ground-borne vibrations due to dynamic loadings from moving trains in subway tunnels," Journal of Zhejiang University (Science A), vol. 13, no. 13, pp. 870-876, 2012.

[8] X. Bian, H. Jiang, C. Chang, J. Hu, and Y. Chen, "Track and ground vibrations generated by high-speed train running on ballastless railway with excitation of vertical track irregularities," Soil Dynamics and Earthquake Engineering, vol. 76, pp. 29-43, 2015.

[9] W. F. Liu, W. N. Liu, S. Gupta, and G. Degrande, "Dynamic response in tunnel and free field due to moving underground trains," Journal of Vibration and Shock, vol. 27, no. 5, pp. 81-84, 2008.

[10] S. Gupta and G. Degrande, "Modelling of continuous and discontinuous floating slab tracks in a tunnel using a periodic approach," Journal of Sound and Vibration, vol. 329, no. 8, pp. 1101-1125, 2010.

[11] M. S. H. Al-Furjan, M. Habibi, L. Shan et al., "On the vibrations of the imperfect sandwich higher-order disk with a lactic core using generalize differential quadrature method," Composite Structures, vol. 257, Article ID 113150, 2021.

[12] M. S. H. Al-Furjan, M. Habibi, G. Chen et al., "Chaotic simulation of the multi-phase reinforced thermo-elastic disk using GDQM," Engineering with Computers, pp. 1-24, 2020.

[13] A. V. Metrikine and A. C. W. M. Vrouwenvelder, "Surface ground vibration due to a moving train in a tunnel: twodimensional model," Journal of Sound and Vibration, vol. 234, no. 1, pp. 43-66, 2000.

[14] M. S. H. Al-Furjan, M. Habibi, D. Won Jung et al., "A computational framework for propagated waves in a sandwich doubly curved nanocomposite panel," Engineering with Computers, pp. 1-18, 2020.

[15] W. T. Thomson, "Transmission of elastic waves through a stratified solid medium," Journal of Applied Physics, vol. 21, no. 2, pp. 89-93, 1950.

[16] D. G. Harkrider, "Surface waves in multilayered elastic medium I: Rayleigh and Love waves from buried sources in a multilayered elastic half-space," Bulletin of the Seismological Society of America, vol. 54, pp. 627-629, 1964.

[17] R. K. N. D. Rajapakse and T. Senjuntichai, "Dynamic response of a multi-layered poroelastic medium," Earthquake Engineering \& Structural Dynamics, vol. 24, no. 5, pp. 703-722, 1995.

[18] T. Senjuntichai and R. K. N. D. Rajapakse, "Exact stiffness method for quasi-statics of a multi-layered poroelastic medium," International Journal of Solids and Structures, vol. 32, no. 11, pp. 1535-1553, 1995.

[19] J. E. Luco and R. J. Apsel, "On the Green's functions for a layered half-space," Bulletin of the Seismological Society of America, vol. 73, pp. 909-929, 1983.

[20] B. Xu, J. F. Lu, and J.-H. Lu, "Dynamic response of an infinite beam overlying a layered poroelastic half-space to moving loads," Journal of Sound and Vibration, vol. 306, no. 1-2, pp. 91-110, 2007.

[21] S. Chen, "Nonlinear dynamics research of vibration of viscoelastic structures," Dissertation, Guangxi University of Science and Technology, Liuzhou, China, 2015.

[22] M. Gu, "Investigation of constitutive relation of viscoelatic material," Journal of Guilin Institute of Electronic Technology, vol. 1, pp. 77-85, 1991. 
[23] C. J. Liu, "Dynamic modeling and analysis of viscoelasticcomposite beams," Dissertation, Northeastern University, Boston, MA, USA, 2011.

[24] G. Suire and G. Cederbaum, "Periodic and chaotic behavior of viscoelastic nonlinear (elastic) bars under harmonic excitations," International Journal of Mechanical Sciences, vol. 37, no. 7, pp. 753-772, 1995.

[25] J. Argyris, V. Belubekian, N. Ovakimyan, and M. Minasyan, "Chaotic vibrations of a nonlinear viscoelastic beam," Chaos, Solitons \& Fractals, vol. 7, no. 2, pp. 151-163, 1996.

[26] L. Q. Chen, C. J. Cheng, and N. H. Zhang, "A dynamic model for nonlinear large-defection viscoelastic beams and its simplification," Shanghai Journal of Mechanics, vol. 3, pp. 302-305, 1999.

[27] L. Q. Chen and C. J. Cheng, "Dynamical behavior of nonlinear viscoelastic beams," Applied Mathematics and Mechanics, vol. 9, pp. 897-902, 2000.

[28] K. Marynowski and T. Kapitaniak, "Kelvin-Voigt versus Bürgers internal damping in modeling of axially moving viscoelastic web," International Journal of Non-linear Mechanics, vol. 37, no. 7, pp. 1147-1161, 2002.

[29] J. Q. Li and H. Z. Liu, "High precision integration for dynamic response of viscoelastic composite structure," Journal of Machine Design, vol. 10, pp. 8-9, 2003.

[30] M. S. H. Al-Furjan, M. Habibi, J. Ni et al., "Frequency simulation of viscoelastic multi-phase reinforced fully symmetric systems," Engineering with Computers, pp. 1-17, 2020.

[31] B. Li, Y. Q. Tang, and H. Ding, "Nonlinear vibrations of axially moving viscoelastic Timoshenko beams under strong external excitation," Journal of Vibration and Shock, vol. 31, no. 13, pp. 142-146, 2012. 